\title{
SOME REMARKS ON THE HASSE NORM THEOREM
}

\author{
HANS OPOLKA
}

ABSTRACT. A substitute for the Hasse norm theorem in Galois extensions of number fields is made more explicit.

1. Let $k$ be a finite extension of $\mathbf{Q}$, let $\bar{k}$ be an algebraic closure of $k$ and let $K \subset \bar{k}$ be a finite Galois extension of $k$ with Galois group $G=G(K / k)$. According to A. Scholz the abelian group

$$
\Re=\Re(K / k)=\frac{\left\{a \in k^{*} \mid a \text { is a norm locally everywhere in } K\right\}}{\left\{a \in k^{*} \mid a \text { is a global norm in } K\right\}}
$$

is called the number knot of $K / k$. It is known to be finite. In $[4,6]$ it is shown that there is a solution of $\Re$, i.e. there is a Galois extension $L$ of $k$ containing $K$ such that $G(L / K)$ is contained in the center of $G(L / k)$ and such that the following modified norm principle holds:

If $x \in k^{*}$ is a norm locally everywhere in $L$ then $x$ is a global norm in $K$.

In this note we ask for the minimum $m(\Re)$ of the set $\{L: K \mid L$ is a solution of $\Re\}$ and the following result will be proved.

THEOREM. Let $K / k$ be a finite Galois extension of number fields with number knot $\Omega$ and let $r$ denote the rank of $\Re$. Then $m(\Re) \leqslant(K: k)^{r}$.

Some remarks are added in the last section.

I am grateful to the referee for his suggestions.

2. We shall use Tate's cohomological description of $\Omega$, see [1, p. 198], namely

$\mathfrak{R}$ is dual to the kernel $\mathfrak{G}=\mathfrak{G}(K / k)$ of the localization map

$$
H^{2}\left(G, \mathbf{C}^{*}\right) \rightarrow \coprod_{v} H^{2}\left(G_{\bar{v}}, \mathbf{C}^{*}\right),
$$

$\mathrm{C}^{*}$ with trivial action, $v$ runs over all places of $k, G_{\bar{v}}$ denotes the decomposition group of some extension $\bar{v}$ of $v$ to $K$.

The following statement is easy to prove, see [6, §2].

$A$ central extension $L$ of $K / k$ is a solution of $\Re$ iff $\mathfrak{Q}$ becomes trivial under inflation $H^{2}\left(G, \mathrm{C}^{*}\right) \rightarrow H^{2}\left(G(L / k), \mathrm{C}^{*}\right)$.

Following I. Schur we shall construct for every cohomology class $\bar{f} \in \mathfrak{G}$ an abstract central group extension $1 \rightarrow B \rightarrow \tilde{G} \rightarrow G \rightarrow 1$ which satisfies the following conditions: $\bar{f}$ becomes trivial under inflation $H^{2}\left(G, \mathbf{C}^{*}\right) \rightarrow H^{2}\left(\tilde{G}, \mathbf{C}^{*}\right),|B|=K: k$,

Received by the editors April 15, 1981 and, in revised form, September 23, 1981.

1980 Mathematics Subject Classification. Primary 12A10, 12A65.

Key words and phrases. Algebraic numbers, Hasse's norm theorem. 
the corresponding embedding problem is solvable. If $L_{f}^{-}$is a solution of this embedding problem then the compositum $L$ of all $L_{\bar{f}}, \bar{f}$ runs over a basis of $\mathscr{\mathcal { G }}$, is a solution of $\mathfrak{B}$ which satisfies $L: K \leqslant(K: k)^{r}$.

3. So take $\bar{f} \in \mathfrak{G}$ and let $m=$ order of $\bar{f}, n=K: k$. Since $\mathbf{C}$ is algebraically closed we may represent $\bar{f}$ by a 2-cocycle $f: G \times G \rightarrow C^{*}$ which takes all its values in $W_{m}$, the group of all $m$ th roots of unity. $W_{m}$ is a subgroup of $B:=W_{n}$ and $f: G \times G \rightarrow B$ defines a central extension $1 \rightarrow B \rightarrow \tilde{G} \rightarrow G \rightarrow 1$ such that $|B|=K: k$ and $\bar{f}$ becomes trivial under inflation.

4. In order to show that the corresponding embedding problem is solvable we shall use a criterium of Hoechsmann [3]: Let $A$ be a finite $G$-module and let $\&=G(\bar{k} / k)$. The group $\hat{A}:=\operatorname{Hom}\left(A, \bar{k}^{*}\right)$ becomes a $\$ S-$-module under the action $\chi^{3}(a)=$ $\left(\chi\left(a^{z^{-1}}\right)\right)^{\mathfrak{z}}, \chi \in \hat{A}, a \in A, z \in \mathbb{B}$. Let $\mathbb{S}_{\chi}$ be the fixed group of $\chi \in \hat{A}$. Then $\chi$ : $A \rightarrow \bar{k}^{*}$ is a $\mathbb{S}_{\chi}$-homomorphism. Consider the following composition of maps:

$$
\bar{\chi}: H^{2}(G, A) \stackrel{\inf }{\rightarrow} H^{2}(\mathbb{S}, A) \stackrel{\text { res }}{\rightarrow} H^{2}\left(\mathscr{S}_{\chi}, A\right) \stackrel{\chi^{*}}{\rightarrow} H^{2}\left(\mathscr{S}_{\chi}, \bar{k}^{*}\right) .
$$

So for $\bar{\varepsilon} \in H^{2}(G, A)$ the image $\bar{\chi}(\bar{\varepsilon})$ defines an element in the Brauer group of the fixed field $k_{\chi}$ of $\mathscr{S}_{\chi}$. Combining Hoechsmann [3, p. 88 and p. 96] with Neukirch [5, p. 80], we get the following

(4.1) Proposition. If $A$ is a trivial G-module then the embedding problem given by $\bar{\varepsilon} \in H^{2}(G, A)$ is solvable if $\bar{\chi}(\bar{\varepsilon})$ splits locally everywhere for all $\chi \in \hat{A}$.

5. Now we apply (4.1) in the situation of $\S 3$, i.e. $A=B=W_{n}, \bar{f} \in \mathfrak{Q}, \varepsilon=f$. Let $\chi \in \hat{B}$ be of order $n$. Then $k_{\chi}=k(\xi)$, where $\xi$ is a primitive $n$th root of unity. Since the restriction $\bar{f}_{\bar{v}}$ of $\bar{f}$ to any decomposition group $G_{\bar{v}}$ is trivial there exists a function $\beta: G_{\bar{v}} \rightarrow C^{*}$ such that $f_{\bar{v}}(x, y)=\beta(x) \beta(y) / \beta(x y)$ for all $x, y \in G_{\bar{v}}$. This implies that the Brauer class of $\bar{\chi}(\bar{\varepsilon})_{\bar{v}}$ can be represented by a 2-cocycle $f_{\chi, \bar{v}}$, such that all values of $f_{\xi, \bar{v}}$ are roots of unity of order dividing $m$ and such that $f_{\xi, \bar{v}}(x, y)=$ $\alpha(x) \alpha(y) / \alpha(x y)$ for all $x, y \in G\left(K_{v}(\xi) / k_{v}(\xi)\right)$, where $\alpha$ is some function on $G\left(K_{v}(\xi) / k_{v}(\xi)\right)$ with values in $\bar{k}_{\bar{v}}^{*}$. Since $f_{\chi, \bar{v}}^{m}=1$ the function $\alpha^{m}$ is a character. So for $e=\left|G_{\bar{v}}\right|$ we have $\alpha^{m \cdot e}=1$. But $m \cdot e$ divides $n$ because

$$
H^{2}\left(G, \mathrm{C}^{*}\right) \stackrel{\text { res }}{\rightarrow} H^{2}\left(G_{\bar{v}}, \mathrm{C}^{*}\right) \stackrel{\text { cor }}{\rightarrow} H^{2}\left(G, \mathrm{C}^{*}\right)=G: G_{\bar{v}}
$$

Hence $\alpha^{n}=1$ and it follows that $\bar{\chi}(\bar{\varepsilon})$ splits at $v$. A similar argument applies to all powers of $\chi$. The proof of our theorem is therefore complete.

6. Some final remarks. (a) In general $|\Re| \leqslant m(\Re)$ (obvious), but one can extract examples from Scholz [7, p. 229], for which $|\Re| \leqslant m(\Re)$ and $m(\Re)=(K: k)^{r}$.

(b) Using the same method as above one can show that $m(\Re)=|\Re|$ if $k$ contains a primitive root of unity of order $K: k$, see [6, (3.2)].

(c) Let $n$ be between $\exp \Re$ and $K: k$ and denote by $K^{\prime}$ the maximal abelian extension of $k$ contained in $K$. A refined version of the proof of the theorem yields the following result. 
We have $m(\Omega) \leqslant n^{r}$ if a primitive nth root of unity $\xi$ is a norm locally everywhere in $K^{\prime}(\xi) / k(\xi)$.

(d) Instead of trying to find the minimum degree of a solution of the number knot of a Galois extension $K / k$ one can ask for a solution with small conductor. This question leads to genus theory. The following result may serve as an example.

Let $K / \mathbf{Q}$ be some finite Galois extension. If $x \in \mathbf{Q}^{*}$ is a norm locally everywhere in the narrow central Hilbert class field $H_{+}$of $K$ then $x$ is a global norm in $K$.

A proof of it is implicit in [4, §4]. In the notation of [4] take $k=\mathbf{Q}, \mathfrak{m}=P_{\infty}=$ the archimedean prime of $\mathbf{Q}$. The only unit in $Z$ which is $\equiv 1\left(P_{\infty}\right)$ is 1 , a global norm from any extension. A slightly sharper variant of this is:

Let $K / \mathbf{Q}$ be some finite Galois extension. If $x \in \mathbf{Q}^{*}$ is a norm locally everywhere in $K$ and if it is a norm locally in $\mathrm{H}^{+}$at the infinite places and at those places where it is a nonunit then it is a global norm in $K$.

This is true because in an unramified local extension a local unit is a norm of a local unit.

\section{REFERENCES}

1. J. W. S. Cassels and A. Fröhlich, Algebraic number theory, Academic Press, London and New York, 1967.

2. D. Garbanati, The Hasse norm theorem for 1-extensions of the rationals, Number Theory and Algebra (Ed., H. Zassenhaus), Academic Press, London and New York, 1977, pp. 77-90.

3. K. Hoechsmann, Zum Einbettungsproblem, J. Reine Angew Math. 229 (1968), 81-106.

4. F. Lorenz, Über eine Verallgemeinerung des Hasseschen Normensatzes, Math. Z. 173 (1980), 203-210.

5. J. Neukirch, Über das Einbettungsproblem der algebraischen Zahlentheorie, Invent. Math. 21 (1973), $59-116$.

6. H. Opolka, Zur Auflösung zahlentheoretischer Knoten, Math. Z. 173 (1980), 95-103.

7. A. Schloz, Totale Normenreste, die keine Normen sind, als Erzeuger nichtobelscher Körpererweiterungen. II, J. Reine Angew Math. 182 (1940), 217-234.

Mathematisches Institut, Roxeler Strasse 64, D-4400 MUNSTer, Germany 